\title{
GENERALIZED CONFORMAL WELDING
}

\author{
D. H. Hamilton
}

\begin{abstract}
Let $\Phi$ be a homeomorphism of the unit circle $\mathbf{T}$ onto itself. Suppose that for any Borel set $G \subset \mathbf{T}$ with $\operatorname{dim} G=0$ we have measure $m_{1}(\Phi(G))=m_{1}\left(\Phi^{-1}(G)\right)=0$. We prove that the unit disk $\mathbf{D}$ and its exterior $\mathbf{D}^{*}$ are mapped by conformal mappings $f$ and $f^{*}$ onto disjoint domains $\Omega$ and $\Omega^{*}$, respectively, so that the radial boundary values satisfy
\end{abstract}

$$
(f \circ \Phi)(z)=f^{*}(z)
$$

for $z \in T-E$, where $m_{1}(E)=0$.

\section{Introduction}

A homeomorphism $\Phi$ of the unit circle $\mathbf{T}=\{|z|=1\}$ onto itself is conformally welded in the the classical sense if the unit circle $\mathbf{D}$ and its exterior $\mathbf{D}^{*}$ may be mapped by some conformal mappings $f$ and $f^{*}$ into disjoint Jordan domains $\Omega$ and $\Omega^{*}$, such that

$$
f^{*}=f \circ \Phi
$$

holds on $\mathbf{T}$. The present paper generalizes the concept of conformal welding to the case where $\Omega$ and $\Omega^{*}$ are not necessarily Jordan domains. It follows from a result of Beurling and Ahlfors, see [4], that any quasi-symmetric $\Phi$ is conformally welded, see Lehto and Virtanen [10], Pfluger [14]. Lehto [9] and David [5] prove conformal welding for other classes of homeomorphisms. One problem is that there exists $\Phi$ for which there is no Jordan curve $\alpha$, e.g.

$$
\Phi\left(e^{i \theta}\right)= \begin{cases}e^{i \pi(\theta / \pi} & 0<\theta<\pi, \\ e^{-i \pi(-\theta / \pi)^{b}} & -\pi<\theta<0,\end{cases}
$$

and $0<a<b<1$. For counterexamples see Oikawa [13], Huber [8], Semmes [16] and Bishop [2], [3].

Nevertheless Bers has asked when is some form of conformal welding possible. This is important for the uniformization of Riemann surfaces. In a companion paper [6] we introduced a generalized conformal welding. The use of Fuchsian groups made it more specialized than the general results we now develop.

We measure sets $E$ by using $p$-Hausdorff measures $m_{p}(E), 0 \leq p \leq 1$. A set $E$ has dimension 0 if $m_{p}(E)=0$ for all $p>0$.

1991 Mathematics Subject Classification: 30C60 
Definition 1. A homeomorphism $\Phi: \mathbf{T} \rightarrow \mathbf{T}$ is regular if for every $E \subset \mathbf{T}$ with $\operatorname{dim} E=0$ we have $m_{1}(\Phi(E))=m_{1}\left(\Phi^{-1}(E)\right)=0$.

Examples. It is easy to see that any homeomorphism $\Phi$ of the Lusin class, i.e., for each

$$
G \subset \mathbf{T}, \quad m_{1}(G)=0 \quad \text { implies } \quad m_{1}(\Phi(G))=m_{1}\left(\Phi^{-1}(G)\right)=0,
$$

is regular. Another example is "Bihölder" homeomorphisms, i.e., there exist positive constants $k, \alpha$ :

$$
k^{-1}\left|z_{1}-z_{2}\right|^{-\alpha}<\left|\Phi\left(z_{1}\right)-\Phi\left(z_{2}\right)\right| \leq k\left|z_{1}-z_{2}\right|^{\alpha},
$$

for all $z_{1}, z_{2} \in \mathbf{T}$. This includes all quasisymmetric maps.

Beurling, see [15], proved that any conformal map $f$ on $\mathbf{D}$ has radial boundary values

$$
f\left(e^{i \theta}\right)=\lim _{r \rightarrow 1} f\left(e^{i \theta}\right)
$$

except for a set of dimension zero. Thus for any regular homeomorphism $\Phi, f \circ \Phi$ is defined on $\mathbf{T}$ (a.e). Our conformal maps will always be bounded on $\mathbf{T}$. Thus one can regard $f \circ \Phi=f^{*}$ as an identity in $L^{\infty}(\mathbf{T})$. We prove

Theorem 1. Let $\Phi: \mathbf{T} \rightarrow \mathbf{T}$ be a regular homeomorphism. Then there exists conformal maps $f, f^{*}$ on $\mathbf{D}, \mathbf{D}^{*}$ respectively so that

(i) $f(\mathbf{D}) \cap f^{*}\left(\mathbf{D}^{*}\right)=\emptyset$,

(ii) $(f \circ \Phi)(z)=f^{*}(z)$, for all $z \in \mathbf{T}-E$, where $m_{1}(E)=0$.

\section{Background results}

Our results depend on the theory of the "fractional-derivative capacity" as well as conformal and quasiconformal mapping.

2.1. Capacity. Maz'ya (Chapter 7 [12]) is the reference for this section. For $u \in C_{0}^{\infty}\left(\mathbf{R}^{2}\right)$ and vanishing in $\{|z|>2\}$, let $\hat{u}$ denote the Fourier transform. For $0 \leq p \leq 1$ define a norm

$$
\|u\|_{p}=\left[\iint|z|^{2 p}|\hat{u}|^{2} d m_{2}\right]
$$

i.e., the $L^{2}$ norm of the $p^{\text {th }}$ order fractional derivative. The space $\mathcal{D}_{p}$ is the closure of $C_{0}^{\infty}\left(\mathbf{R}^{2}\right)$ (vanishing in $\{|z| \geq 2\}$ ) in the $\|\cdot\|_{p}$ norm. The capacity $C_{p}$ is defined for compact sets $E \subset\{|z| \leq 1\}$ by

$$
C_{p}(E)=\inf \left\{\|u\|_{p}: u \in \mathcal{D}_{p}, u \geq 1 \text { on } E\right\} .
$$


This is indeed a capacity in the sense of Choquet, essentially coinciding with the logarithmic capacity for $p=1$.

The first property we need is:

(i) For $p<q, \mathcal{D}_{q}$ is compactly embedded in $\mathcal{D}_{p}$, i.e., suppose we have a sequence $u_{n} \in \mathcal{D}_{q}$ with $\left\|u_{n}\right\|_{q} \leq 1$. Then there is a subsequence $u_{n_{k}}$ and $u \in \mathcal{D}_{q}$ so that

$$
\left\|u_{n_{k}}-u\right\|_{p} \rightarrow 0
$$

It follows from the definition and property (i) that: For any sequence $u_{n} \in \mathcal{D}_{q}$ with $\left\|u_{n}\right\|_{q} \leq 1$, any $p<q$, there is a set $E, C_{p}(E)<\varepsilon$,

$$
u_{n_{k}} \rightarrow u(z)
$$

uniformly on $\mathbf{R}^{2}-E$.

We also make use of the relation between $p$-capacity and Hausdorff dimension.

(ii) For any $0<p \leq 1, E \subset \mathbf{R}^{2}$

$$
\operatorname{dim} E>2-2 p \quad \text { implies } \quad C_{p}(E)>0 .
$$

We apply these results to functions $h(z)=\sum_{k=1}^{\infty} b_{k} z^{k}$ analytic on $\mathbf{D}$ with

$$
\iint_{D}\left|h^{\prime}\right|^{2} d x d y=\pi \sum_{k=1}^{\infty} k\left|b_{k}\right|^{2}<\infty
$$

By Beurling, see [15],

$$
\lim _{r \rightarrow 1} h\left(r e^{i \theta}\right)
$$

exists and is finite except for $e^{i \theta} \in E$, with $C_{1}(E)=0$. We refer to this limit as $h\left(e^{i \theta}\right)$ (when it exists). (In general, $h(z)$ may be extended to $u \in \mathcal{D}_{1}$.)

Applying (i), (ii) immediately yields:

Theorem 2. Let $h(z)=\sum_{k=1}^{\infty} a_{k, n} z^{k}$ be analytic on $\mathbf{D}$ with $\sum_{k=1}^{\infty} k\left|a_{k, n}\right|^{2}$ $\leq 1$. Then there exists a subsequence $h_{n_{k}}$ and a limit $h(z)$ so that for every $p<1$ and $\varepsilon>0$ there is a set $E \subset \mathbf{T}$ with $C_{p}(E) \leq \varepsilon$ and

$$
h_{n_{k}}(z) \rightarrow h(z)
$$

uniformly on $\mathbf{T}-E$.

2.2. Conformal mapping. We shall be considering pairs $\left\{f, f^{*}\right\}$ where $f$ is conformal on $\mathbf{D}$ and $f^{*}$ is conformal on $\mathbf{D}^{*}$. It is important that $f(\mathbf{D}) \cap$ 
$f^{*}\left(\mathbf{D}^{*}\right)=\emptyset$. However, as we can just as well consider $\left\{j \circ f, j \circ f^{*}\right\}$ for any Möbius transformation $j$, we shall assume the following normalization:

$$
\begin{aligned}
f^{*}(z) & =z+\sum_{k=1}^{\infty} a_{k} z^{-k}, \quad|z|>1, \\
f(z) & =\sum_{k=1}^{\infty} b_{k} z^{k}, \quad|z|<1 .
\end{aligned}
$$

This implicitly assumes $b_{0} \in f(\mathbf{D}), b_{0} \notin f^{*}\left(\mathbf{D}^{*}\right)$. The classical theory shows that

$$
\left.\begin{array}{c}
\partial f^{*}\left(\mathbf{D}^{*}\right) \\
f(\mathbf{D})
\end{array}\right\} \subset\{|w| \leq 2\},
$$

see Pommerenke [15]. We shall call this the $S$-normalization. Now as $f$ and $f^{*}-z$ may be extended to $\mathcal{D}_{1}$, from Section 2.1 we immediately deduce

Lemma 1. Let $\left\{f_{n}, f_{n}^{*}\right\}$ be a sequence of $S$-normalized pairs. Then there is an analytic function $f$ on $\mathbf{D}, f^{*}$ conformal on $\mathbf{D}^{*}$, and a subsequence $n_{k}$ so that for any $p<1, \varepsilon>0$

$$
f_{n_{k}}^{*}\left(e^{i \theta}\right) \rightarrow f^{*}\left(e^{i \theta}\right), \quad f_{n_{k}}\left(e^{i \theta}\right) \rightarrow f\left(e^{i \theta}\right)
$$

uniformly for $e^{i \theta} \in \mathbf{T}-E, C_{p}(E) \leq \varepsilon$. zero.

Remarks. 1. Thus $f^{*}\left(e^{i \theta}\right) \rightarrow f^{*}\left(e^{i \theta}\right)$ pointwise except for a set of dimension

2. The exceptional set cannot be replaced by one with logarithmic capacity zero.

3. The function $f$ may be identically constant. We make use of a lemma of Beurling (see [15]), to ensure that $f_{n_{k}}$ does not collapse.

Lemma 2. Let $f^{*}$ be conformal on $\mathbf{D}^{*}$. Then for any constant $b$

$$
C_{1}\left\{e^{i \theta}: f^{*}\left(e^{i \theta}\right)=b\right\}=0 .
$$

Remarks. This fails in $\mathcal{D}_{1}$.

2.3. Quasiconformal mappings. References may be found in [11]. Let a homeomorphism $\Phi: \mathbf{T} \rightarrow \mathbf{T}$ be quasisymmetric on $\mathbf{T}$. Beurling and Ahlfors, see [11], proved that $\Phi$ extends to a quasiconformal mapping of $\mathbf{C}$, i.e., $\partial \Phi$ and $\bar{\partial} \Phi \in L^{2}(\mathbf{C})$ and $\|\bar{\partial} \Phi / \partial \Phi\|_{\infty}<1$. Let the quasiconformal map $\Phi^{-1}$ have complex dilatation

$$
\mu(z)=\frac{\bar{\partial} \Phi^{-1}}{\partial \Phi^{-1}}, \quad \text { for } z \in \mathbf{C} .
$$


Bojarski's theorem says that for any measureable $\lambda$ with $\|\lambda\|_{\infty}<1$ there is a quasiconformal homeomorphism $\psi: \mathbf{C} \rightarrow \mathbf{C}$ so that $\partial \psi=\lambda \partial \psi$ (a.e. with respect to area). Applying this to

$$
\lambda(z)= \begin{cases}\mu(z) & z \in \mathbf{D}^{*} \\ 0 & z \in \mathbf{D}\end{cases}
$$

yields a quasiconformal map $f$ with dilatation 0 on $\mathbf{D}$. The composition formulae shows that $f^{*} \equiv f \circ \Phi$ has dilatation 0 on $\mathbf{D}^{*}$. Thus $f$ is conformal on $\mathbf{D}$ and $f^{*}$ is conformal on $\mathbf{D}^{*}$. This is the argument of Lehto and Virtanen [10] or Pfluger [14] for

Lemma 3. For quasisymmetric $\boldsymbol{\Phi}: \mathbf{T} \rightarrow \mathbf{T}$ there are complementary Jordan domains $\Omega, \Omega^{*}$ and conformal maps

$$
f: \mathbf{D} \rightarrow \Omega, \quad f^{*}: \mathbf{D}^{*} \rightarrow \Omega^{*}
$$

so that the boundary values (on $\mathbf{T}$ ) satisfy

$$
f^{*}=f \circ \Phi \text {. }
$$

2.4. Regular homeomorphisms. We now give some basic properties of regular homeomorphisms. First we observe that a regular homeomorphism is absolutely continuous in the sense of:

Definition 2. A homeomorphism $\Phi: \mathbf{T} \rightarrow \mathbf{T}$ is RC ("regularly continuous") if for every $p<1$ and $\varepsilon>0$ there is a $\delta>0$ so that for any $E \subset \mathbf{T}$ with $C_{p}(E)<\delta$, we have

$$
m_{1}(\Phi(E)), m_{1}\left(\Phi^{-1}(E)\right)<\varepsilon .
$$

The proof is given in

Lemma 4. $A$ homeomorphism $\Phi: \mathbf{T} \rightarrow \mathbf{T}$ is $\mathrm{RC}$ if and only if it is regular.

We only show that every regular $\Phi: \mathbf{T} \rightarrow \mathbf{T}$ is RC. Otherwise there is a sequence of $E_{n} \subset \mathbf{T}, C_{p}\left(E_{n}\right) \leq 1 / 2^{n}$, so that $m_{1} \Phi E_{n} \geq \varepsilon>0$, say. Setting $F_{n}=\sum_{k=n}^{\infty} E_{k}$, and as $\operatorname{dim} F_{n} \leq 1 / 2^{n-1}$ we obtain $F_{n} \supset F_{n+1} \supset \cdots$ with $m_{1} \Phi F_{n} \geq \varepsilon$. Thus $F_{n} \downarrow \lim F_{n} \equiv F$ with $\operatorname{dim} F=0$ but $m_{1} \Phi(F) \geq \varepsilon$ by monotone convergence.

We cannot approximate a regular homeomorphism $\Phi: \mathbf{T} \rightarrow \mathbf{T}$ by smooth $\Phi_{n}$ which are "uniformly RC". However a one sided approximation is possible.

Lemma 5. Let $\Phi_{n}: \mathbf{T} \rightarrow \mathbf{T}$ be a regular homeomorphism with

$$
\omega(\delta, p)=\sup \left\{m_{1} \Phi^{-1}(E): C_{p}(E) \leq \delta\right\} .
$$

Then there exists a sequence of smooth homeomorphisms $\Phi_{n}: \mathbf{T} \rightarrow \mathbf{T}$ :

(i) $\sup \left\{m \Phi_{n}^{-1}(E): C_{p}(E) \leq \delta\right\} \leq \omega(\delta, p)$,

(ii) $k_{n}^{-1}|z-w| \leq\left|\Phi_{n}(z)-\Phi_{n}(w)\right| \leq k_{n}|z-w|$ for all $z, w \in \mathbf{T}$, with constants $k_{n}$

(iii) $\Phi_{n}^{-1}(z) \rightarrow \Phi^{-1}(z), \Phi_{n}(z) \rightarrow \Phi(z)$ uniformly on $T$. 
This result is only about $\Phi^{-1}$ which we write as

$$
\Phi^{-1}\left(e^{i \theta}\right)=e^{i \varphi(\theta)}
$$

where $\varphi: \mathbf{R} \rightarrow \mathbf{R}$ is a homeomorphism so that for all integers $k$

$$
\varphi(\theta+2 \pi k)=\varphi(\theta)+2 \pi k .
$$

Up to a constant the $p$-capacity on $\mathbf{T}$ is equivalent to the obvious capacity for $\mathbf{R}$. Clearly $\varphi$ is "RC", i.e. for any $\varepsilon>0$ there is a $\delta>0$ so that for any $E \subset[0,2 \pi], C_{p}(E)<\delta$,

$$
m_{1} \varphi(E)<\varepsilon .
$$

Let $\tau_{n} \in C^{\infty}$ be an approximation to the identity

(i) $\tau_{n}(x)>0$ on $[-1 / n, 1 / n]$,

(ii) $\tau_{n}(x)=0,|x| \geq 1 / n$,

(iii) $\int_{-\infty}^{\infty} \tau_{n}(x) d x=1$.

We set

$$
\varphi_{n}(x)=\int_{x-1 / n}^{x+1 / n} \tau_{n}(y) \varphi(x+y) d y .
$$

Clearly $\varphi_{n}$ is a smooth homeomorphism with $\varphi_{n}(x+2 \pi k)=\varphi_{n}(x)+2 \pi k$. Also for any $E \subset[0,2 \pi), C_{p}(E) \leq \delta$,

$$
\begin{aligned}
m_{1}\left(\varphi_{n}(E)\right) & =\int_{E} d \varphi_{n}=\iint_{\mathbf{R}} \tau_{n}(y) d y d x \\
& =\int_{\mathbf{R}} \tau_{n}(y) \int_{E} d \varphi(x+y) d x d y \\
& =\int_{\mathbf{R}} \tau_{n}(y) m_{1}(\varphi(E+y)) d y \leq \omega_{p}(\delta) .
\end{aligned}
$$

Thus setting $\Phi_{n}^{-1}\left(e^{i \theta}\right)=e^{i \varphi_{n}(\theta)}$ we obtain a homeomorphism with the required properties.

\section{Proof of Theorem 1}

Let $\Phi: \mathbf{T} \rightarrow \mathbf{T}$ be a regular homeomorphism. We use the approximating homeomorphisms $\Phi_{n}$ constructed in Lemma 5. These quasisymmetric maps are extended to $\mathbf{C}$ and as in 2.2 we obtain maps $f_{n}, f_{n}^{*}$ conformal on $\mathbf{D}, \mathbf{D}^{*}$ respectively so that

$$
f_{n}^{*}(z)=f_{n} \circ \Phi_{n}(z)
$$

for $z \in \mathbf{T}$. We assume that $\left\{f_{n}, f_{n}^{*}\right\}$ are $S$-normalized and thus that $f_{n}^{*}, f_{n}$ converge normally on $\mathbf{D}^{*} \cup \mathbf{D}$ to $f^{*}, f$ (which may be constant). Observe that 
$f^{*}, f \circ \Phi$ are well defined functions of $L^{\infty}(\mathbf{T})$. Let $p$ be any polynomial in $z, \bar{z}$. Now

$$
\int_{\mathbf{T}} p(z) f_{n}^{*}(z)|d z| \rightarrow \int_{\mathbf{T}} p(z) f^{*}(z)|d z|
$$

by normal convergence. The left hand side is equal to

$$
\int p(z) f_{n} \circ \Phi_{n}(z)|d z|=\int_{\mathbf{T}} p\left(\Phi_{n}^{-1}(w)\right) f_{n}(w)\left|d \Phi_{n}^{-1}\right|
$$

where $w=\Phi_{n}(z)$. Now for any $\delta>0$ and $p<1$ there is a set $E$ with $C_{p}(E)<\delta$ and $f_{n}(w) \rightarrow f(w)$ uniformly on $\mathbf{T}-E$. For any $\varepsilon>0$, for small enough $\delta$,

$$
\int_{E}\left|d \Phi_{n}^{-1}\right|<\varepsilon
$$

by the results of Sections 2.1-2.4. Thus as $p \circ \Phi_{n}^{-1} \rightarrow p \circ \Phi^{-1}$ uniformly on $\mathbf{T}-E$ :

$$
\int_{\mathbf{T}-E} p\left(\Phi_{n}^{-1}(w)\right) f_{n}(w)\left|d \Phi_{n}^{-1}\right| \rightarrow \int_{\mathbf{T}-E}\left(p \circ \Phi^{-1}\right) f(w)\left|d \Phi^{-1}\right|
$$

Now as $p, f_{n}$ are bounded on $E$

$$
\left|\int_{E} p\left(\Phi_{n}^{-1}(w)\right) f_{n}(w)\right| d \Phi_{n}^{-1}||<C \varepsilon
$$

Combining these results implies

$$
\int_{\mathbf{T}} p(z) f_{n} \circ \Phi_{n}(z)|d z| \rightarrow \int_{\mathbf{T}}\left(p \circ \Phi^{-1}\right) f(w)\left|d \Phi^{-1}\right| .
$$

Now as $\Phi$ is regular and $f$ may be approximated uniformly (except for a set of small capacity) by continuous functions, we change variable of integration to obtain

$$
\int_{\mathbf{T}} p(z)(f \circ \Phi)(z)|d z|=\int_{\mathbf{T}} p(z) f^{*}(z)|d z| .
$$

Therefore $f \circ \Phi=f^{*}$ in $L^{\infty}(\mathbf{T})$.

Finally observe that $f$ cannot be constant, as otherwise so is the conformal mapping $f^{*}$, by Lemma 2 . 


\section{Uniqueness of representation}

Let $q$ : $\mathbf{C} \rightarrow \mathbf{C}$ be any homeomorphism which is conformal on $\Omega \cup \Omega^{*}$, so that $q(z)=z+\sum_{k=1}^{\infty} c_{k} z^{-k}$ near $\infty$. Then if $\left\{f, f^{*}\right\}$ is a ( $S$-normalized) conformal welding for a homeomorphism $\Phi: \mathbf{T} \rightarrow \mathbf{T}$ we obtain a second ( $S$-normalized) conformal welding $\left\{q \circ f, q \circ f^{*}\right\}$.

Thus even in the classical case that $\Omega, \Omega^{*}$ are the inner and outer domain of a closed Jordan curve $\alpha$ there may exist such a $q$. This is obvious if Area $\alpha>0$ for then we define dilatation $\mu$ on $\alpha$ and let $q$ be the normalized quasiconformal solution of the Beltrami equation

$$
\bar{\partial} q=\mu \partial q .
$$

However there exists examples of nonuniqueness even when Area $\alpha=0$ (but $\operatorname{dim} \alpha>1$ ), see Bishop [2]. Our example is constructed by classification results of Ahlfors and Beurling.

Let $\Omega$ be any domain containing $\infty$ with complement $E$. The Dirichlet class $\mathcal{D}(\Omega)$ is the set of functions

$$
h(z)=\sum_{k=1}^{\infty} a_{k} z^{-k}, \quad(|z|>R)
$$

analytic on $\Omega$ with

$$
\|h\|=\sqrt{\int_{\Omega}\left|h^{\prime}\right|^{2} d x d y}<\infty .
$$

The capacity with respect to $\mathcal{D}(\Omega)$ is

$$
c(E)=\sup \left\{\left|a_{1}\right|: h \in \mathcal{D}(\Omega),\|h\| \leq 1\right\} .
$$

Then there are nonmöbius conformal maps defined on $\Omega$ if and only if $c(E)>0$.

Now let $E$ be a totally disconnected compact set. Let $\Omega, \Omega^{*}$ be disjoint simply connected domains with $\infty \in \Omega^{*}$ and constructed so that

$$
\partial \Omega=\partial \Omega^{*} \supset E .
$$

By scaling and translation we may assume that the conformal maps

$$
f: \mathbf{D} \rightarrow \Omega, \quad f^{*}: \mathbf{D}^{*} \rightarrow \Omega^{*}
$$

are $S$ normalized.

We can construct $\Omega$ and $\Omega^{*}$ by a family of Jordan arcs connecting components of $E$. Thus we may define a map

$$
\Phi=f^{-1} \circ f^{*}
$$


defined at first only on $\mathbf{T}-F$, where $f^{*}(F)=E$. By suitable construction of $\Omega$, $\Omega^{*}$ one can ensure that $\operatorname{dim} F>0$ and $\Phi$ extends to a regular homeomorphism of $\mathbf{T}$. Thus $\left\{f, f^{*}\right\}$ is a generalized conformal welding of $\Phi$.

Now provided $c(E)>0$ there is a nonmöbius conformal map $q$ of $\mathbf{C}-E$ (not necessarily a homeomorphism of $\mathbf{C}$ ). We may assume that $q$ is normalized. Thus we obtain a pair $\left\{q \circ f, q \circ f^{*}\right\}$ which forms a conformal welding in our generalized sense.

\section{Non Jordan case}

Now we discuss the case that $\partial \Omega$ (or $\partial \Omega^{*}$ ) is not a Jordan curve. The example in the introduction cannot be conformally welded in the classical sense. Our proof of Theorem 1 shows that there is a dense subset $F \subset \partial \Omega=\partial \Omega^{*}$ so that every $z \in F$ is the endpoint of open Jordan $\operatorname{arcs} \beta \subset \Omega, \beta^{*} \subset \Omega^{*}$. Furthermore $f^{-1}(F)$ is a dense subset of $\mathbf{T}$ (of positive dimension). Thus if $\lim _{r \rightarrow 1} f\left(r e^{i \theta}\right)$ does not exist there is then a nontrivial cluster set $\chi$ of $f$ at $e^{i \theta}$. In prime-end theory (see [15]) $\chi$ is the impression associated with $e^{i \theta}$. The arcs $\beta, \beta^{*}$ separate these continua so that each $e^{i \theta}$ maps to a unique impression $I\left(e^{i \theta}\right)$. Thus for a regular homeomorphism $\Phi$

$$
\Phi=f^{*} \circ f^{-1}
$$

in the sense of prime-ends.

As soon as there is a single nontrivial continua $\chi=I\left(e^{i \theta}\right)$ then the conformal welding of $\Phi$ by $\left\{f, f^{*}\right\}$ is not unique. Any normalized conformal map $q$ of $\mathbf{C}-\chi$ gives a conformal welding of $\Phi$ by $\left\{q \circ f, q \circ f^{*}\right\}$.

Note that we can always find a conformal mapping $q$ so that $q(\chi)$ is a horizontal line segment. In the next section we observe that there is always a conformal welding so that all the impressions $\chi$ are horizontal line segments.

\section{Class of representations}

For each regular homeomorphism $\Phi: \mathbf{T} \rightarrow \mathbf{T}$ let $\mathcal{F}_{\Phi}$ be the class of pairs $\left\{f, f^{*}\right\}$ of ( $S$-normalized) conformal maps

$$
f: \mathbf{D} \rightarrow \Omega, \quad f^{*}: \mathbf{D}^{*} \rightarrow \Omega^{*},
$$

$\Omega \cap \Omega^{*}=\emptyset, f^{*}\left(e^{i \theta}\right)=(f \circ \Phi)\left(e^{i \theta}\right)$ (a.e. on $\left.\mathbf{T}\right)$. The following is an immediate deduction from Section 2 :

Lemma 6. $\mathcal{F}_{\Phi}$ is compact in the topology of uniform convergence on compact subsets of $\mathbf{D} \cup \mathbf{D}^{*}$.

Theorem 3. For every regular homeomorphism $\Phi: \mathbf{T} \rightarrow \mathbf{T}$ there is a conformal welding $\left\{f, f^{*}\right\}$ so that

(i) Area $\left(\mathbf{C}-\left\{f(\mathbf{D}) \cup f^{*}\left(\mathbf{D}^{*}\right)\right\}\right)=0$,

(ii) Each impression of $\partial f(\mathbf{D})$ (and $\left.\partial f^{*}\left(\mathbf{D}^{*}\right)\right)$ is a horizontal line segment. 

$\in \mathcal{F}_{\Phi}$

On $\mathcal{F}_{\Phi}$ consider the problem of maximizing $\operatorname{Re} a_{1}$ for $f^{*}(z)=z+\sum_{k=1}^{\infty} a_{k} z^{-k}$

Now for each pair

$$
f^{*}=z+\sum_{k=1}^{\infty} a_{k} z^{-k}, \quad f=\sum_{k=0}^{\infty} b_{k} z^{k}
$$

of $\mathcal{F}_{\boldsymbol{\Phi}}$ we have the area formulae

$$
\operatorname{Area}\left(\mathbf{C}-\left\{f(\mathbf{D}) \cup f^{*}\left(\mathbf{D}^{*}\right)\right\}\right)=\pi-\pi \sum_{k=1}^{\infty} k\left(\left|a_{k}\right|^{2}+\left|b_{k}\right|^{2}\right), \quad a_{0}=0
$$

Thus

$$
1 \geq \sum_{k=0}^{\infty} k\left(\left|a_{k}\right|^{2}+\left|b_{k}\right|^{2}\right)
$$

Regarding $\mathcal{F}_{\Phi}$ as a bounded subset of the obvious Hilbert space we see that $\left\{f^{*}, f\right\} \in \mathcal{F}_{\Phi}$ is an extreme point if and only if

$$
\text { Area }\left(\mathbf{C}-\left\{f(\mathbf{D}) \cup f^{*}\left(\mathbf{D}^{*}\right)\right\}\right)=0 .
$$

Now by Lemma 6 there exists an extremal $f \in \varepsilon \times \tau\left(\mathcal{F}_{\Phi}\right)$ maximizing $\operatorname{Re} a_{1}$. Thus we may assume the existence of an extremal satisfying (i).

Now let $\chi$ be any impression of $\partial f(\mathbf{D})$ which is not a horizontal line segment. By the variational theory of Schiffer, see [15], there is a function

$$
g(z)=z+\sum_{k=1}^{\infty} d_{k} z^{-k}
$$

conformal on $\mathbf{C}-\chi$, so that $\operatorname{Re} d_{1}>0$. Consider the pair $\left\{g \circ f^{*}, g \circ f\right\} \in \mathcal{F}_{\Phi}$. Then if $g \circ f^{*}=z+\sum_{k=1}^{\infty} e_{k} z^{-k}$ as $e_{1}=d_{1}+a_{1}$, we get $\operatorname{Re} e_{1}>\operatorname{Re} a_{1}$ which is a contradiction.

Remarks. Using quasiconformal variations one can prove every extremal satisfies (i).

\section{References}

[1] Ahlfors, L.V., and L. Bers: Riemann's mapping theorem for variable metrics. - Ann. Math. 72, 1960, 385-404.

[2] Bishop, C.: A counterexample in conformal welding concerning Hausdorff dimension. Mich. Math. J. 35, 1988, 151-159.

[3] Bishop, C.: Conformal welding of rectifiable curves. - Preprint. 
[4] Douady, A., and C.J. EARLE: Conformally natural extension of homeomorphisms of the circle. - Acta. Math. 157, 1986, 23-48.

[5] David, G.: Solutions de l'equation de Beltrami avec $\|\mu\|_{\infty}=1$. - Ann. Acad. Sci. Fenn. Ser. A I Math. 13, 1988, 25-70.

[6] Hamilton, D.H.: Simultaneous uniformization. - To appear.

[7] Huber, A.: Konforme Verheftung von Gebieten mit beschrenkter Randdrehung. - Comment. Math. Helv. 50, 1975, 179-186.

[8] Huber, A.: Isometrische und konforme Verheftung. - Comment. Math. Helv. 51, 1976, 319-331.

[9] Lehto, O.: Homeomorphisms with given dilatation. - Proceedings of the 15th Scandinavian Congress, Oslo 1968. Lecture Notes in Mathematics 118, Springer-Verlag, 1970.

[10] Lehto, O., and K. VirTanen: On the existence of quasiconformal mappings with prescribed dilatation. - Ann. Acad. Sci. Fenn. Ser. A I Math. 274, 1960.

[11] Lehto, O., and K. VirTANEn: Quasiconformal mappings in the plane. - Springer-Verlag, 1973.

[12] MAZ'YA, V.G.: Sobolev spaces. - Springer Series in Soviet Mathematics, Springer-Verlag, 1985.

[13] OikaWA, K.: Welding of polygons and the type of a Riemann surfaces. - Kodai Math. Sem. Rep. 13, 1961, 37-52.

[14] Pfluger, A.: Über die Konstruktionen Riemannscher Flächen durch Verheftung. - J. Indian Math. Soc. 24, 1960, 401-412.

[15] Pommerenke, C.: Univalent functions. - Vandenhoeck \& Ruprecht, 1975.

[16] Semmes, S.W.: A counterexample in conformal welding concerning chord arc curves. Ark. Math. 24, 1986, 141-158.

[17] Vainio, J.V.: Conditions for the possibility of conformal sewing. - Ann. Acad. Sci. Fenn. Ser. A I Math. Dissertationes 53, 1985.

University of Maryland

Department of Mathematics

College Park, MD 20742

U.S.A.

Received 21 December 1990 